\title{
RADIO-WAVE TOMOGRAPHY OF INHOMOGENEITIES IN BIOLOGICAL MEDIA WITH MULTI-FREQUENCY SOUNDING IN THE RANGE 2-8 GHZ
}

\author{
Sergey Shipilov ${ }^{1,1}$ Aleksandr Eremeev $^{1}$, Evgeny Balzovsky ${ }^{1}$, Elena Yurchenko $^{2}$ \\ ${ }^{1}$ National Research Tomsk State University, the Department of Radiophysics, 634050 Tomsk, Russia \\ ${ }^{2}$ National Research Tomsk Polytechnic University, 634050, Tomsk, Russia
}

\begin{abstract}
In this paper, a method for detecting and mapping inhomogeneities in biological tissues using the radio-wave tomosynthesis method is presented. The proposed method of radio-wave tomosynthesis allows us to calculate the three-dimensional distribution of the permittivity of the space under study and, thereby, to detect tissue inhomogeneities and to determine their location and size. Due to their harmlessness for humans, these methods are suitable for dynamic observation of changes in the size of formation, in contrast to x-ray methods, for which regular doses of ionizing radiation are contraindicated. Therefore, the development of non-invasive methods for the search for inhomogeneities in biological media based on radio-wave sounding, which makes it possible to identify pathological formations, is now very relevant.
\end{abstract}

\section{Introduction}

To date, due to the development of information sciences and computer technology, it has become possible to use the methods of radio-wave tomography as the means of remote nondestructive testing and diagnostics of the internal structure, semitransparent media for radio emission and restoration of the form of opaque objects. There is a great need for contactless and automated medical diagnostics. The best means for such tasks is radio wave tomography.

At present, ultrasonic scanners and x-ray tomographs are used to detect tumors in biological tissues, but it is proposed to use a radio-wave tomograph for earlier and safe control of the appearance of neoplasms. In pathological and physiological processes in biological tissues, as a rule, there are changes in their electrophysical properties, and measurement task of which is of considerable interest in various medical applications. This is important for the detection of cancer. When neoplasms appear, they accumulate liquid and blood, that leads to a change in the permittivity of the given formation $[1,2]$. A change in the dielectric constant can be detected using methods of radio wave tomography [3, 6-8]. Due to their harmlessness to humans, these methods are suitable for constant monitoring of changes in the size of formation, in contrast to x-ray methods, for which regular doses of ionizing radiation are

\footnotetext{
${ }^{1}$ Corresponding author: s.shipilov@gmail.com
} 
contraindicated. Therefore, the development of non-invasive methods for the search for inhomogeneities in biological media based on radio-wave sounding, which makes it possible to identify pathological formations, is now very relevant.

\section{Measurement of the permittivity of a biological medium}

In this paper, to solve the problem of tomography of inhomogeneities in biological medium is used the known method of radio-wave tomosynthesis [3]. For its application it is necessary to know the permittivity of the medium. With the help of methods and a coaxial measuring cell developed at the department of radiophysics of Tomsk State University, it is possible to calculate the dielectric constant of a biological material as measured in a wide frequency range.

Figure 1 shows a coaxial measuring cell.

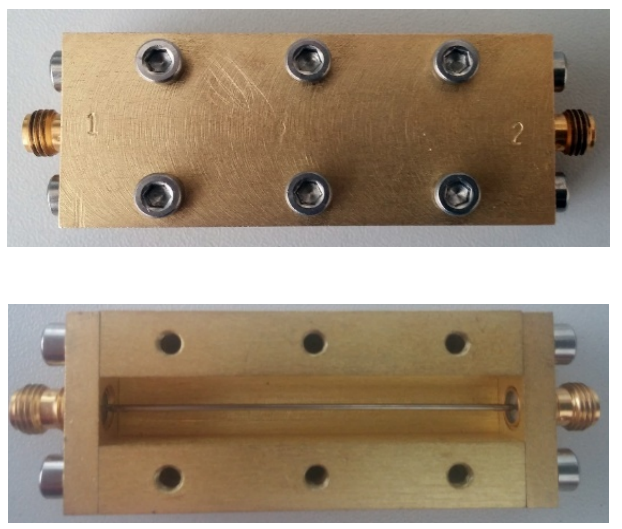

Fig. 1. Coaxial measuring cell.

To measure the complex dielectric constant in the $10 \mathrm{MHz}-8 \mathrm{GHz}$ frequency range, a coaxial line measurement method was used which was successfully used to measure the dielectric properties of liquids and bulk materials [4, 5]. This method is based on measuring the transmission and reflection coefficients of a section of a coaxial line. This method is characterized by a large broadband and comparative simplicity of processing the results of measurements. It makes it possible to measure the complex dielectric permittivity of samples, with both low and high dielectric permittivity and dielectric losses. Figure 2 shows the measurements. The investigated medium was placed in the cell, which is the section of the coaxial line with the wave resistance of $50 \mathrm{Ohm}$ and the length of $5 \mathrm{~cm}$. Electromagnetic waves of the given frequency were passed through the cell with matter. Pork melted fat was used as a biological medium. 


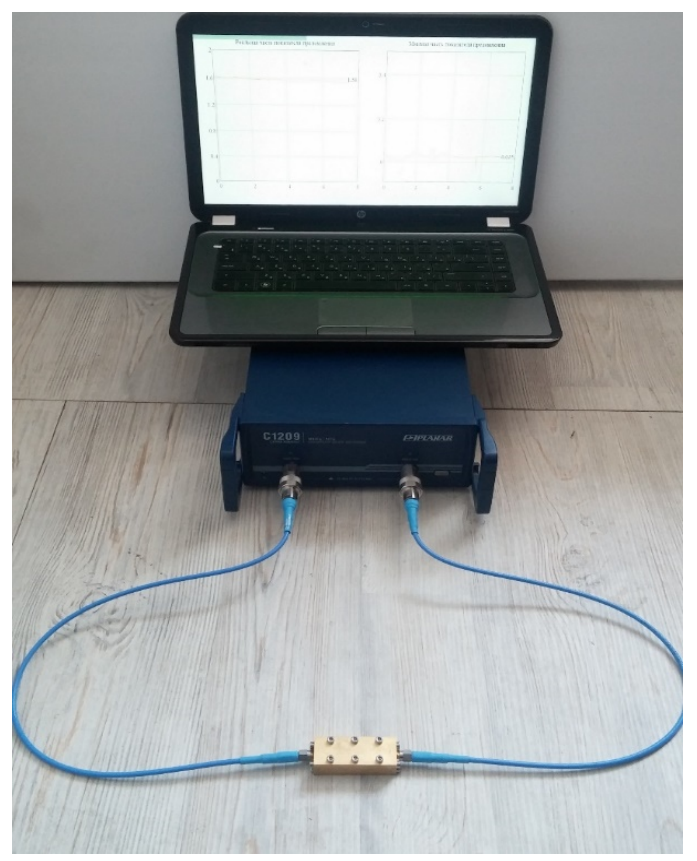

Fig. 2. Measurement of the permittivity of a biological medium.

The components of the scattering matrix of the coaxial measuring cell - complex reflection (parameters S11, S22) and transmission (parameters S12, S21) coefficients - were measured using "Planar" vector of network analyzer C1209.

Figure 3 shows the results of measurements - the dependence of the real and imaginary parts of the refractive index of pork fat on frequency at room temperature.

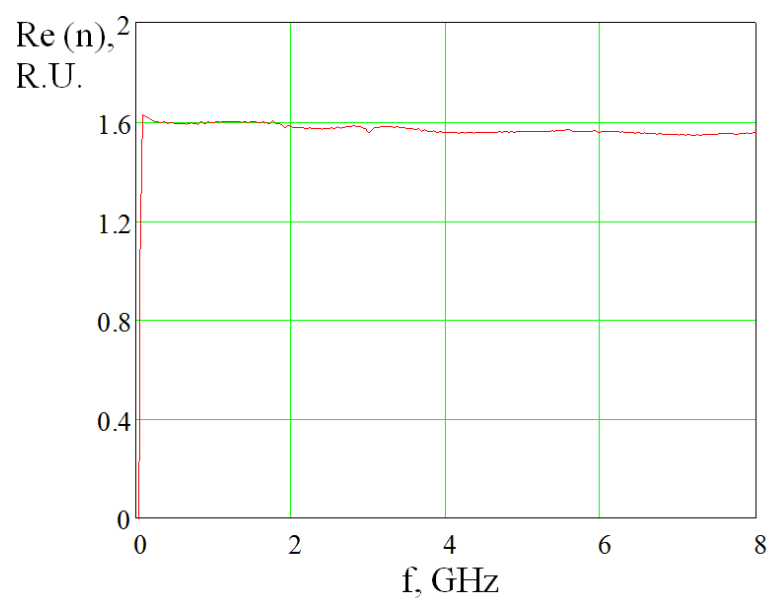

a) 


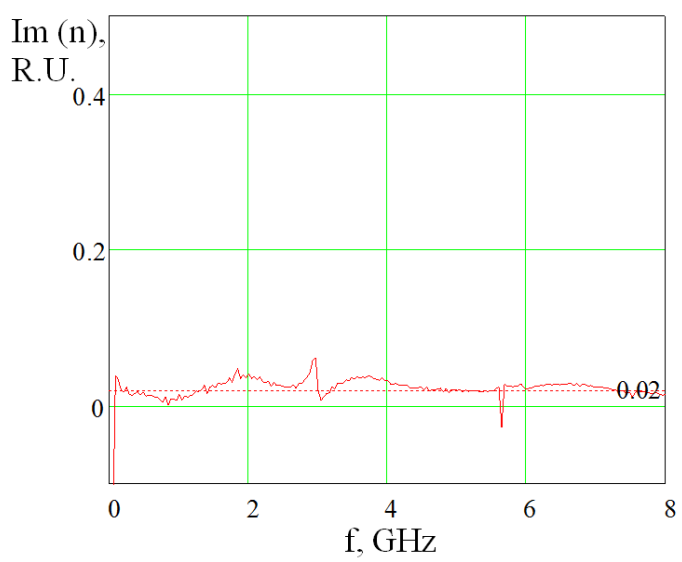

b)

Fig. 3. Dependence of the refractive index of pork fat on frequency: a-real part, b-imaginary part.

A decrease in the real part of the complex dielectric constant with increasing frequency was observed. The results obtained are in good agreement with the results known from the literature.

\section{Experiment on the detection of inhomogeneities in the biological medium}

The measurements were carried out in the frequency range 2-8 GHz. To carry out these experiments to search for inhomogeneities in a biological medium, a measuring device was used. This device consisted of the vector reflectometer "Caban R140" with ultra-wideband (UWB) antenna (operating frequency range 1.5-8.5 GHz) [9]. Figure 4 shows the scheme and the photo of the experiment.
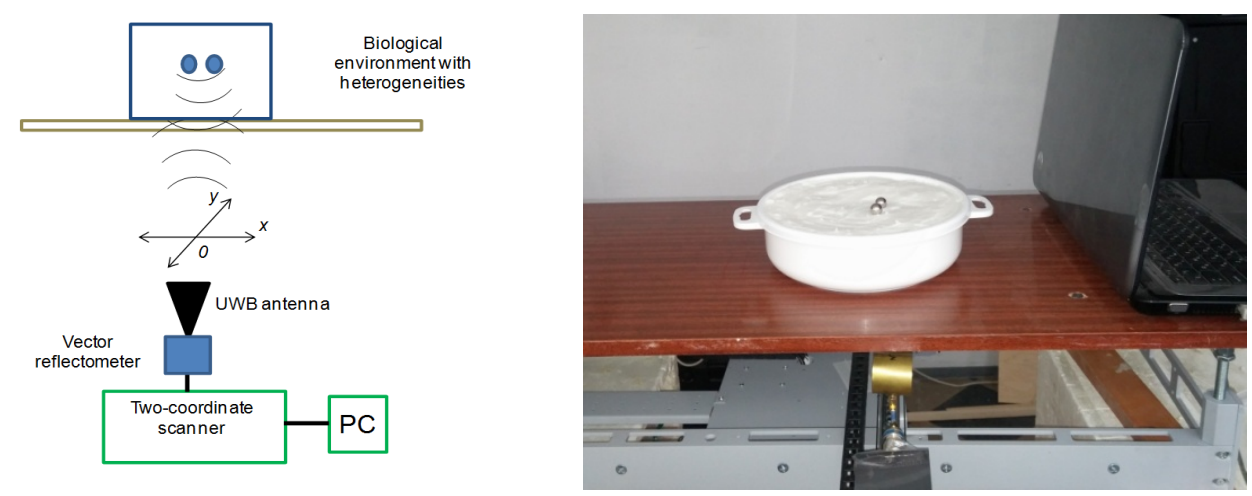

Fig. 4 Scheme of the experiment on the detection of inhomogeneities in the biological medium and its photograph.

The measuring device was fixed on a moving carriage of a two-coordinate scanner for obtaining multi-angle projections of a probable biological medium with heterogeneities hidden 
in it. Heterogeneities were test objects in the form of metal and plastic balls filled with saline solution. The diameter of the balls was $1.3 \mathrm{~cm}$. The distances between the centers of the balls were $3 \mathrm{~cm}$. Pork fat which is close to the human in its electrodynamic properties was used as a background biological medium.

The images of artificial heterogeneities in the biological medium were obtained after the experiment. Figure 5 shows the results of the experiments.
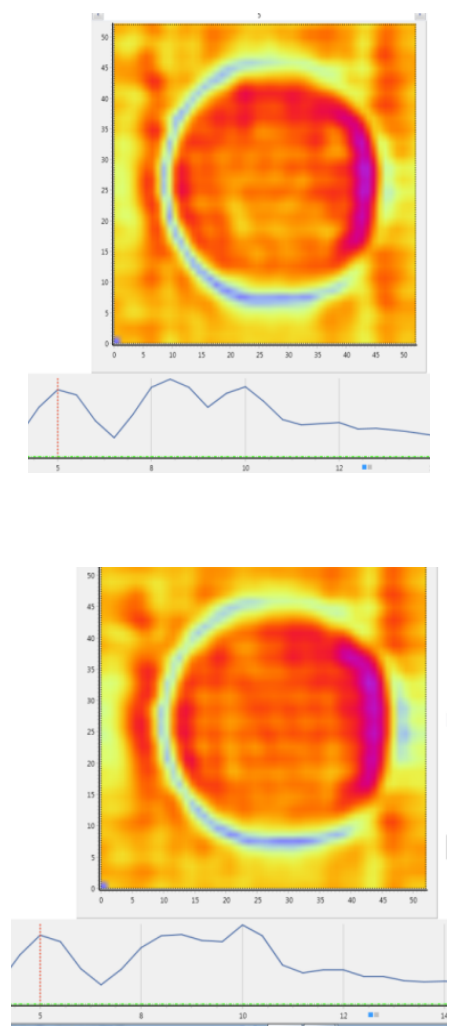

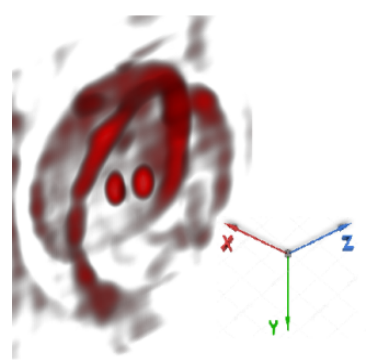

a)
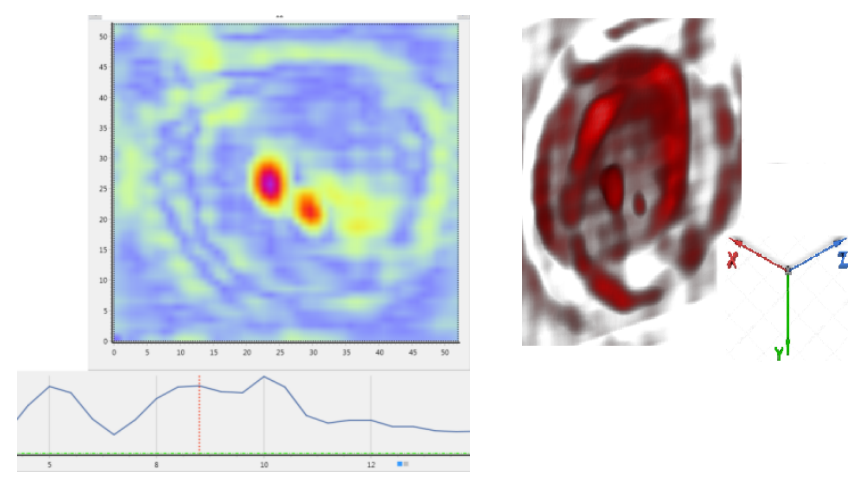

b)

Fig. 5 The result of tomography of the biological environment: a - metal balls, b-plastic balls filled with saline solution.

Thus, the results obtained make it possible to construct a three-dimensional tomogram of the distribution of inhomogeneities in a biological medium.

\section{Conclusion}

In this paper, the method for detecting and mapping inhomogeneities in biological tissues using the radio-wave tomosynthesis method is presented. The proposed method of radio-wave tomosynthesis makes it possible to calculate the three-dimensional distribution of the permittivity of the space under study and, thereby, to detect tissue inhomogeneities and to determine their location and size.

Based on the results of the experiments, it is possible to estimate the resolving power not worse than $2 \mathrm{~cm}$ when using the frequency range of 2-8 GHz for probing. 


\section{Acknowledgements}

The research was supported by the Russian Ministry of Education and Science as a part of the state order No 3.2068.2017/4.6.

\section{References}

[1] M. Lazebnik, D. Popovic, L. McCartney, C.B. Watkins, M.J. Lindstrom, J. Harter, S. Sewall, T. Ogilvie, A. Magliocco, T.M. Breslin, W. Temple, D. Mew, J.H. Booske, M. Okoniewski, S.C. Hagness, Phys. Med. Biol. 52, 6093 (2007)

[2] M. Lazebnik, E.L. Madsen, G.R. Frank, S.C. Hagness, Phys. Med. Biol. 50, 4245 (2005)

[3] S.E. Shipilov, R.N. Satarov, V.P. Yakubov, A.V. Yurchenko, O.V. Minin, I.V. Minin, Optical and Quantum Electronics, Optical and Quantum Electronics 49, 339 (2017)

[4] A.G. Gorriti, E.C. Slob, IEEE TGRS 43, 2051 (2005)

[5] J. Baker-Jarvis, E.J. Vanzura, W.A. Kissick, IEEE Trans. Microw. Theory Tech. 38, $1096(1990)$

[6] A.V. Klokov, A.S. Zapasnoy, A.S. Miron'Chev, E.A. Dyukarev, S.V. Smirnov, S.S. Shipilova, IOP J. Phys: Conf. Series 881, 012013 (2017)

[7] A.V. Klokov, A.S. Zapasnoy, A.S. Miron'Chev, V.P. Yakubov, S.S. Shipilova, IOP J. Phys: Conf. Series 671, 012028 (2016)

[8] D. Sukhanov, K. Zavyalova, MATEC Web of Conferences 79, 01040 (2016)

[9] E. Balzovsky, Yu. Buyanov, MATEC Web of Conferences 79, 01036 (2016) 